\title{
Layer-by-layer crystallization and the role of fluctuations in free standing smectic films
}

\author{
Andrea Fera, ${ }^{1}$ Boris I. Ostrovskii, ${ }^{2}$ Daniel Sentenac, ${ }^{1}$ Ivan Samoilenko, ${ }^{2}$ and Wim H. de Jeu ${ }^{1}$ \\ ${ }^{1}$ FOM-Institute for Atomic and Molecular Physics, Kruislaan 407, 1098 SJ Amsterdam, The Netherlands \\ ${ }^{2}$ Institute of Crystallography, Academy of Sciences of Russia, Leninsky pr. 59, Moscow 117333, Russia
}

(Received 28 June 1999)

\begin{abstract}
Specular x-ray reflectivity has been used to study the changes in the thermal fluctuation behavior of eight layer freely suspended $N$-(4-n-butoxybenzilidene)-4-n-octylaniline (4O.8) films during the process of successive layer-by-layer crystallizations. Each of these steps is preceded by the formation of an intermediate layer structure with in-plane hexatic ordering. We find an unusually large reduction of the fluctuations after the emergence of only the first hexatic toplayer. The fluctuation profiles over the film are in all cases quenched at the surfaces though less so after each crystallization step. This behavior supports theoretical arguments that hexatic order is reduced by out-of-plane smectic layer fluctuations. [S1063-651X(99)51111-2]

PACS number(s): 61.10.Kw, 64.70.Md, 68.35.Rh
\end{abstract}

In the smectic- $A(\mathrm{Sm}-A)$ liquid crystalline phase the elongated molecules have their long axis on average parallel to a preferred direction in space (director $\mathbf{n}$ ). In addition the centers of mass are arranged in equidistant layers perpendicular to $\mathbf{n}$, while the system remains fluid in the plane of the layers. The reduced dimensionality of smectic liquid crystals leads to strong thermal fluctuations of the smectic layers. As a consequence the positional ordering of the layers is not truly long range: the mean-square displacement of the layers $\left\langle u^{2}(\mathbf{r})\right\rangle$ diverges with the sample size (Landau-Peierls instability) [1]. Upon cooling a $\mathrm{Sm}-A$ phase, a hexatic Sm- $B$ phase may occur that shows long-range bond-orientational order while positional order is limited to a finite correlation length [1]. At lower temperatures a three-dimensional (3D) ordered crystalline $\mathrm{B}(\mathrm{CrB})$ phase may be found with an hexagonal in-plane lattice. Sm- $A$ liquid crystals can be suspended over an opening in a solid frame. Such free standing films have a high degree of uniformity and a controlled thickness ranging from two to over hundreds of layers [2]. They provide a unique opportunity to study phase transitions in substrate-free finite-size systems, showing a crossover from $3 \mathrm{D}$ behavior in thick films to $2 \mathrm{D}$ behavior in thin ones. These observations have inspired to search in freely suspended smectic films for the true nature of the KosterlitzThouless transition, which describes 2D melting as a twostage process proceeding via the unbinding of topological defects [1].

In liquid crystals a free surface may stabilize a higher ordered phase that is only observed at lower temperatures in the bulk or not observed in the bulk at all [3]. Such "surface crystallization' is outside the field of liquid crystals also found in some long chain alkanes and alcohols [4]. Here we want to concentrate on the compound usually indicated as 4O.8 N-(4- $n$-butoxybenzilidene)-4- $n$-octylaniline [see Fig. 1(a)]. In free standing films of 40.8 Pindak and co-workers [5] originally demonstrated the $3 \mathrm{D}$ crystalline nature of the $\mathrm{CrB}$ phase from the solidlike shear response of a lowfrequency torsional oscillator in contact with the film. From the shear response two anomalies were detected: at the Sm- $A-\mathrm{CrB}$ bulk transition and about $6^{\circ}$ above this temperature. Later Jin, Stoebe, and Huang [6] measured via ac calorimetry on 40.8 films a whole cascade of phase transitions, attributed to step-by-step surface crystallization. Subsequently electron diffraction studies by Ho et al. [7] provided conclusive evidence for layer-by-layer crystallization from $\mathrm{Sm}-A$ into $\mathrm{CrB}$ mediated via an hexatic $\mathrm{Sm}-B$ phase not observed in the bulk. Interestingly the directions of the hexatic, respectively crystalline lattice in the top layers at both sides of a film appear to be correlated, though there are liquid $\mathrm{Sm}-A$ layers in between them.

In spite of the results described, some important questions still remain open. (i) What is the mechanism driving the cascade of subsequent transitions? (ii) How do the correlated directions between lattices in well-separated toplayers arise? These problems are of course related to the general interest whether these layer transitions are true examples of a Kosterlitz-Thouless process. In this Rapid Communication we provide at least a partial answer to these questions by investigating eight layer 40.8 films using x-ray reflectivity. This provides insight into the changes in the fluctuation profiles in dependence of the number of crystallization steps. The observed behavior supports theoretical arguments [8] that the hexatic order is reduced by the out-of plane smectic layer fluctuations, which means in our situation enhanced at the surfaces compared to the interior of the film.

The compound investigated, 4O.8, [Fig. 1(a)], was obtained from Aldrich and was purified additionally via several recrystallization steps. Freely suspended smectic films were drawn and mounted in a two-stage oven and equilibrated as described earlier [9]. From rocking curves the final mosaicity

\section{K $33 \mathrm{CrB} 48.5 \mathrm{SmA} 63.5 \mathrm{~N} 78 \mathrm{I}$}

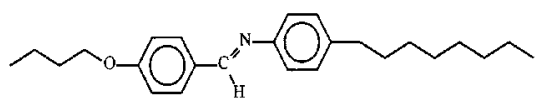

(a)
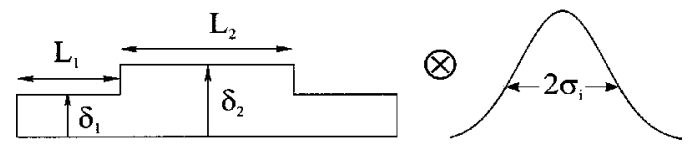

(b)

FIG. 1. (a) Structural formula of 40.8 and its bulk phase transition temperatures $\left({ }^{\circ} \mathrm{C}\right)$. (b) Box model (see text). 
of the films was determined to be typically of the order of $0.01^{\circ}$ over the footprint of the beam. In a specular reflectivity experiment an incident beam of wavelength $\lambda$ and wave number $k=2 \pi / \lambda$ is reflected at an interface. For elastic scattering, $\left|\mathbf{k}_{f}\right|=\left|\mathbf{k}_{i}\right|=k$, while the incident wave vector $\mathbf{k}_{i}$, the reflected wave vector $\mathbf{k}_{f}$ and the surface normal lie in the same (scattering) plane. Consequently, the resulting momentum transfer $|\mathbf{q}|=\left|\mathbf{k}_{f}-\mathbf{k}_{i}\right|=\mathbf{q}_{z}=2 k \sin \alpha$ is parallel to the surface normal. Thermally excited layer fluctuations scatter part of the beam away from the specular direction and cause deviations from the Fresnel reflectivity of a smooth interface. In the case of a film, reflection also occurs at the second interface, leading to constructive or destructive interference in dependence of the incoming angle (Kiessig fringes). The period of the Kiessig fringes is inversely proportional to the film thickness $D$. In smectic films the internal periodic structure generates in addition finite size broadened Bragg-like peaks centered at $q=2 \pi \mathrm{m} / d$, where $d$ is the layer spacing and $m$ an integer. Hence the number of smectic layers in the film $N=D / d$ is immediately determined from the specular diffraction profile. Specular reflectivities were taken at beamline BW2 of HASYLAB (DESY, Hamburg) using a sixcircle diffractometer at $k=45.5 \mathrm{~nm}^{-1}$. In the scattering plane, the divergence of the incident beam, $\Delta \alpha$, and the detector acceptance, $\Delta \beta$, were both fixed by slits. A direct measurement of $\Delta \alpha$ using narrow predetector slits gave a value of $0.064^{\circ}$, while the total resolution was of the order of $0.076^{\circ}$. In the out-of-plane direction the slits were set to a width of $2 \mathrm{~mm}$. All curves are background subtracted and have been corrected for geometrical effects as described earlier [9].

Figure 2(a) shows specular scans for an eight layer film in the high-temperature $\mathrm{Sm}-A$ phase and the final $\mathrm{CrB}$ phase together with fits to the model to be described. The intensity in the region just after the first Bragg peak is most sensitive to the development of the crystalline top-layers. Changes in the shape of the electron density profile through the film are reflected by the deep minimum appearing at about $2.6 \mathrm{~nm}^{-1}$. Figure 2(b) displays a blow-up of different specular reflectivity scans of this film at different temperatures. Starting from the $\mathrm{Sm}-A$ phase, the temperature was lowered at a controlled speed $\left(\sim 0.1{ }^{\circ} \mathrm{C} / \mathrm{min}\right)$ while monitoring changes in the density profile of the film by means of fast specular scans around the first Bragg peak. The scans are different after each crystallization of an additional layer. Furthermore, preceding each transition of a $\mathrm{Sm}-A$ into a $\mathrm{CrB}$ layer, in the region between 2.5 and $2.7 \mathrm{~nm}^{-1}$ small but very reproducible changes in the intensity were detected, interpreted as the intermediary formation of a hexatic $\mathrm{Sm}-B$ phase [7]. The equilibration time of each transition is of the order of seconds and no influence of the cooling speed has been detected. In this way the transition temperatures of the various layers could be determined with an accuracy of the order of $0.2^{\circ} \mathrm{C}$. They are displayed in Fig. 3 and are in good agreement with literature indications [7a].

The data have been fitted with an iterative matrix solution of the Fresnel equations for the reflectivity of the multilayer system using a slab-model for the electron density profiles [9]. Each smectic layer has been approximated by the boxlike function represented in Fig. 1(b). In order to take thermal fluctuations into account, the box-model has been con-
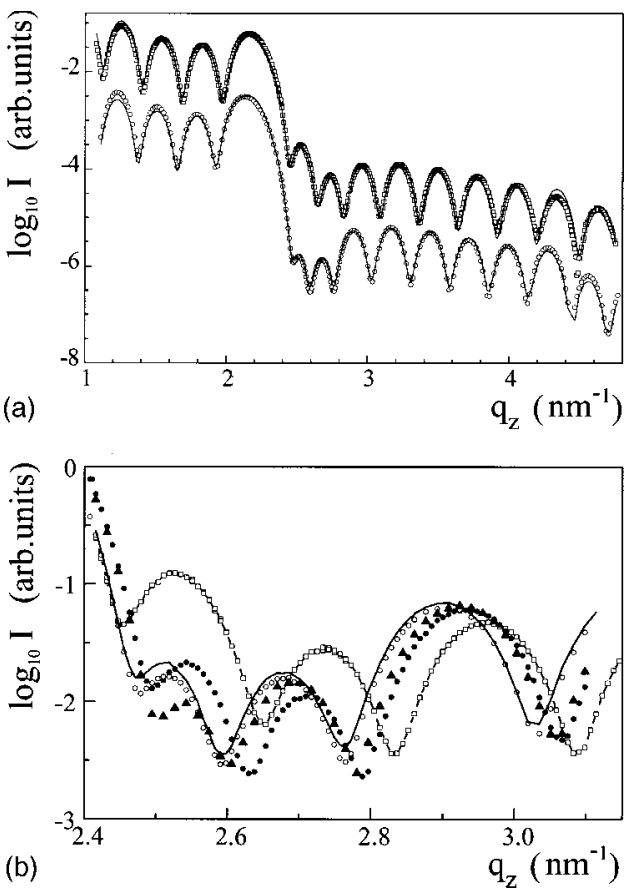

FIG. 2. (a) Specularly reflected $\mathrm{x}$-ray intensity $I$ of an eight layer film in the $\mathrm{Sm}-A$ phase $\left(62.5^{\circ} \mathrm{C}\right.$, upper curve $)$ and in the $\mathrm{CrB}$ phase $\left(47.0^{\circ} \mathrm{C}\right.$, lower curve). The curves have been shifted one decade for clarity; solid line is a fit using the box model. (b) Blow up of the specularly reflected $\mathrm{x}$-ray intensity $I: \quad 62.5^{\circ} \mathrm{C}, \mathrm{Sm}-A$ phase (squares); $52.5^{\circ} \mathrm{C}$, one $\mathrm{CrB}$ top layer (closed circles); $50.3^{\circ} \mathrm{C}$, two $\mathrm{CrB}$ top layers (triangles); $49.65^{\circ} \mathrm{C}$, two $\mathrm{Sm}-A$ layer left in the middle (open circles); $47.0^{\circ} \mathrm{C}, \mathrm{CrB}$ phase (solid line).

voluted with a Gaussian of full width at half maximum (FWHM) equal to $2 \sigma \sqrt{2 \ln 2}$. Each set of data was fitted independently, keeping $L_{i}$ and $\delta_{i}$ fixed for the particular set. In contrast $\sigma_{i}$ was left free for each smectic layer, taking the centro-symmetry of the density profile into account. In the absence of reliable data around the critical angle no absolute values of $\delta_{1}$ and $\delta_{2}$ have been determined. Nevertheless, all calculations converged to $\delta_{2} / \delta_{1} \approx 1.5$ and $L_{2} / L_{1} \approx 2.1$.

The fluctuation profiles over the film are presented in Fig. 4(a); error bars indicate $95 \%$ confidence limits. In the $\mathrm{Sm}-\mathrm{A}$ phase the fluctuations are quenched at the surface. After the first crystallization step appreciable damping occurs, in spite of the fact that only a single $\mathrm{CrB}$ top layer is present that by definition has no $3 \mathrm{D}$ ordering. Upon the next layer crystallization further damping occurs while now the profile is also strongly flattened. Within the error bars this behavior essentially does not change anymore after the third step. Figure 4(b) gives more details about the fluctuations during the transitions in the first top layer. The emergence of a hexatic top

\begin{tabular}{|c|c|}
\cline { 2 - 2 } & $\mathrm{SmA} 61.1 \mathrm{SmB} 54.5 \mathrm{CrB}$ \\
\hline & $\mathrm{SmA} 51.5 \mathrm{SmB} 50.4 \mathrm{CrB}$ \\
\hline & $\mathrm{SmA} 50.1 \mathrm{SmB} 49.7 \mathrm{CrB}$ \\
\hline & $\mathrm{SmA} 49.6 \mathrm{SmB} 48.0 \mathrm{CrB}$ \\
\hline
\end{tabular}

FIG. 3. "Phase diagram" of an eight layer free standing film of 40.8; the layer number is indicated at the left. 

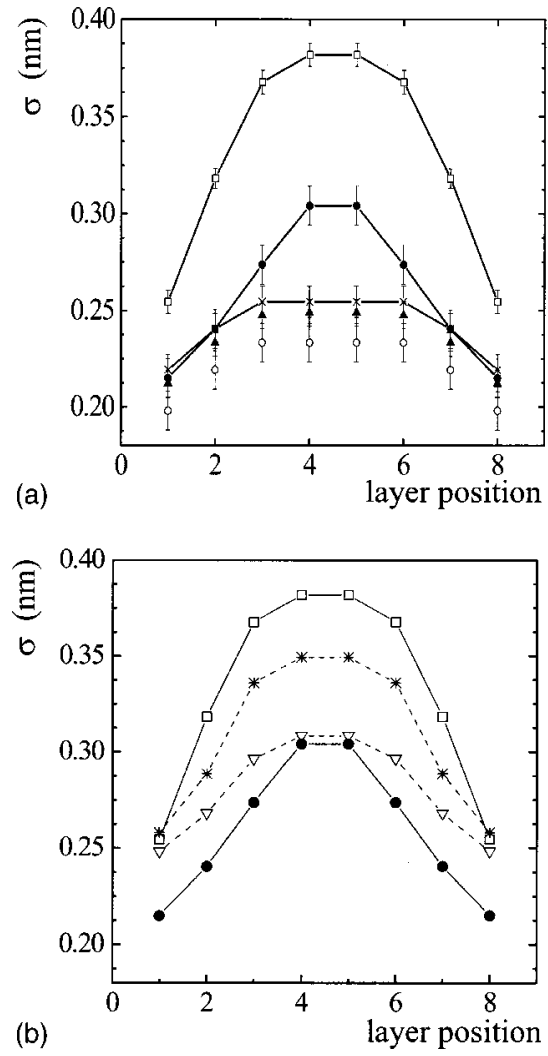

FIG. 4. (a) Fluctuation profiles of an eight layer film: $62.5^{\circ} \mathrm{C}$, $\mathrm{Sm}-A$ phase, (squares); $52.5^{\circ} \mathrm{C}$, one $\mathrm{CrB}$ top layer (closed circles); $50.3{ }^{\circ} \mathrm{C}$, two $\mathrm{CrB}$ top layers (open circles); $49.65^{\circ} \mathrm{C}$, two $\mathrm{Sm}-A$ layers left in the middle (triangles); $47.0^{\circ} \mathrm{C}, \mathrm{CrB}$ phase (crosses). (b) Details of the fluctuation profile of an eight layer film (top layer transitions only): $\mathrm{Sm}-A$ phase at $62.5^{\circ} \mathrm{C}$ (squares); hexatic $\mathrm{Sm}-B$ top layer at $60.7^{\circ} \mathrm{C}$ (asterisks), and $55.7^{\circ} \mathrm{C}$ (inverted triangles); $\mathrm{CrB}$ top layer at $52.5^{\circ} \mathrm{C}$ (closed circles).

layer already leads to a considerable damping of the fluctuations in the film.

Thermal fluctuations in free standing $\mathrm{Sm}-A$ films have been well studied $[9,10]$. They can be described by the Landau-de Gennes theory extended with a surface term for $\mathbf{r}=(\mathbf{R}, z=D / 2)[11]$,

$$
\begin{aligned}
F= & \frac{1}{2} \int d^{3} r\left[B\left(\frac{\partial u(\mathbf{r})}{\partial z}\right)^{2}+K\left[\Delta_{\perp} u(\mathbf{r})\right]^{2}\right] \\
& +\frac{1}{2} \gamma \int d^{2} r\left[\nabla_{\perp} u\left(\mathbf{R}, z= \pm \frac{1}{2} D\right)\right]^{2}
\end{aligned}
$$

The bulk behavior is determined by the elastic constants for bending $\left(K \approx 10^{-11} \mathrm{~N}\right)$ and for compression $\left(B \approx 10^{7} \mathrm{~N} / \mathrm{m}^{2}\right)$ of the layers. The fluctuations are damped at the surface if the ratio $\nu=\gamma / \sqrt{B K}>1$. Taking for 40.8 a value of $\gamma$ $\approx 0.02 \mathrm{~N} / \mathrm{m}$ [12] we arrive at $\nu \approx 2$ in agreement with the quenching of the fluctuations observed at the surfaces in the Sm- $A$ phase (Fig. 4).

Before discussing the layer-by-layer transitions let us consider the final stage when the whole film is in the $\mathrm{CrB}$ phase. Following Ref. [13] the elastic energy of a plate cut from a hexagonal crystal can be calculated. However, as in the $\mathrm{CrB}$ phase the shear elastic constant $\mathrm{C}_{44}$ is at least two orders of magnitude smaller than the other elastic constants [14], the layers can easily glide over each other. Hence, we can apply the approximation that all deformations but the shear ones are forbidden. As a result an additional term $\mathrm{C}_{44}\left[\nabla_{\perp} u(\mathbf{r})\right]^{2}$ must be added to the terms in $B$ and $K$ in Eq. (1) [15]. In Eq. (1) the layer compression term and the surface term do not change appreciably when the $\mathrm{CrB}$ lattice is established. In the easy shear approximation also the bend rigidity of the film is not strongly affected [15]. Hence a $\mathrm{CrB}$ film can still be considered as a fluctuating system, in agreement with the experimental findings. Comparing the $\mathrm{Sm}-A$ and the $\mathrm{CrB}$ phase [Fig. 4(a)] we note a decrease of the fluctuations, which can be attributed to the extra sliding elasticity term connected with $\mathrm{C}_{44}$ and probably some increased values of $B$ and $\gamma$. In addition there is a flattening of the profile over the film due to the diminishing importance of the surface term.

Let us now consider the transitions in the outermost layers [Fig. 4(b)]. Reference [8] considers the first stage of a Kosterlitz-Thouless mechanism (Sm- $A-\mathrm{Sm}-B$ ) driven by the unbinding of disclination pairs. Smectic layer fluctuations are coupled to the hexatic order because of a frustration in the bond-angle field due to the curvature of the smectic layers. As a general result the layer fluctuations decrease the disclination core energy and thus the $\mathrm{Sm}-A-\mathrm{Sm}-B$ transition temperature. Hence if the layer fluctuations in the $\mathrm{Sm}-A$ phase are quenched at the surface (as shown in Fig. 4) the liquid-hexatic transition temperature is correspondingly larger at the surface than in the bulk. We note that in some compounds also the situation $\nu<1$ has been observed [9]. It would be interesting to investigate whether the associated enhanced surface fluctuations lead indeed to conventional surface melting. After the first Sm- $A-\mathrm{Sm}-B$ transition, upon further cooling considerable changes were detected in the fluctuations profiles over the film [see Fig. 4(b)] within the temperature range of the hexatic $\mathrm{Sm}-B$ toplayers. Such a behavior suggests that the second stage of the transition ( Sm- $B-\mathrm{CrB}$ ) probably evolves by a continuous increase of the correlation length for positional order in the hexatic toplayers.

In the early experiments on the hexatic order in the surface layers of 40.8 , it was found that the bond-order directions of top and bottom layers were coupled [7]. This has been considered as a rather unexpected phenomena as the intermediate layers still have the $\mathrm{Sm}-A$ liquid in-plane structure. However, from our previous work on smectic films $[9,10]$ the hydrodynamic fluctuations in the Sm- $A$ phase can be expected to be fully conformal throughout the film. As a consequence any directional interaction between the fluctuation amplitude and the local hexatic lattice will be the same at both sides of the film. Hence conformal fluctuations provide a natural mechanism to correlate lattice directions in the top and bottom surface hexatic layers.

Now let us consider the evolution of the fluctuation profiles in the eight layer film as the crystallization advances. After crystallization of the top layer the remaining inner Sm- $A$ layers still fluctuate, but with a strongly diminished amplitude [Fig. 4(a), filled circles]. The large magnitude of this reduction is surprising, as a single toplayer (whether hexatic Sm-B or $\mathrm{CrB}$ ) should not experience any effect of sliding elasticity yet. In fact the suppression of the fluctuations in the interior of the film is already strong when the 
first surface hexatic layer has developed [Fig. 4(b)]. In agreement with early ultrasound results on a bulk transition $\mathrm{Sm}-A$ to hexatic Sm- $B$ [16], an hexatic surface layer probably modifies the in-plane structure of a neighboring $\mathrm{Sm}-A$ layer by imposing pretransitional ordering. The induced order, in turn, would lead locally to some sliding elasticity and hence to a decrease of the amplitude of the fluctuations. In this interpretation our measurements support speculations along similar lines to explain the reduced Sm- $A-\mathrm{Sm}-B$ transition enthalpy with decreasing thickness of the film [17]. Anyhow, the new profile provides the basis for the second series of crystallization transitions in the next-nearest top layers. Once two layers are crystallized at each side, the fluctuations of the remaining four $\mathrm{Sm}-A$ layers are such that the total profile over the film is much more flat. In agreement with this behavior the differences in transition temperatures between the 'new' $\operatorname{Sm} A$ surface layers and those in the center of the film approach each other rapidly.

In conclusion we have used specular x-ray reflectivity to characterize the process of layer-by-layer crystallization in eight layer freely suspended smectic 40.8 films. The fluctuation profile in the initial Sm- $A$ film is quenched at the sur- faces, supporting theoretical predictions that correlate the onset of hexatic order with decreased layer fluctuations. As the fluctuations can be expected to be fully conformal throughout the film, this process also explains the reported correlation of the crystallographic directions in the top and bottom surface layers. The changing fluctuation behavior in the successive layers triggers off a cascade of successive Sm- $A-\mathrm{Sm}-B-\mathrm{CrB}$ phase transitions per layer. Quantitatively the suppression of the fluctuations in the interior of the film is already important once the first hexatic toplayer is established.

We thank Arcadi Shalaginov (St. Petersburg, Russia) for valuable discussions, R. Eidenschink (Nematel, Mainz, Germany) for recrystallizing 40.8, and Mourits Nielsen (Ris $\emptyset$, Roskilde, Denmark) for his assistance in setting up the measurements at HASYLAB. Work at HASYLAB was supported by Contract No. ERBFMGECT950059 of the European Community. This work is part of the research program of the Stichting voor Fundamenteel Onderzoek der Materie, (FOM), which is financially supported by the Nederlandse Organisatie voor Wetenschappelijk Onderzoek, (NWO).
[1] See, for example, P. M. Chaikin and T. C. Lubensky, Principles of Condensed Matter Physics (Cambridge University Press, Cambridge, England, 1995).

[2] See, for some recent reviews, P. Pieranski et al., Physica A 195, 364 (1993); C. Bahr, Int. J. Mod. Phys. B 8, 3051 (1994).

[3] B. M. Ocko, A. Breslau, P. S. Pershan, J. Als-Nielsen, and M. Deutsch, Phys. Rev. Lett. 57, 94 (1986); E. F. Gramsbergen, W. H. de Jeu, and J. Als-Nielsen, J. Phys. (Paris) 47, 711 (1986); E. B. Sirota, P. S. Pershan, S. Amador, and L. B. Sorensen, Phys. Rev. A 35, 2283 (1987); B. D. Swanson, H. Stragier, D. Tweet, and L. B. Sorensen, Phys. Rev. Lett. 62, 909 (1989); T. Stoebe, R. Geer, C. C. Huang, and J. W. Goodby, ibid. 69, 2090 (1992); C. Y. Chao, S. W. Hui, J. E. Maclennan, C. F. Chou, and J. T. Ho, ibid. 78, 2581 (1997).

[4] O. Gang, B. M. Ocko, X. Z. Wu, E. B. Sirota, and M. Deutsch, Phys. Rev. Lett. 80, 1264 (1998).

[5] D. E. Moncton and R. Pindak, Phys. Rev. Lett. 43, 701 (1979); R. Pindak, D. J. Bishop, and W. O. Sprenger, ibid. 44, 1461 (1980)

[6] A. J. Jin, T. Stoebe, and C. C. Huang, Phys. Rev. E 49, R4791 (1994).

[7] C. Y. Chao, C. F. Chou, J. T. Ho, S. W. Hui, A. Jip, and C. C.
Huang, Phys. Rev. Lett. 77, 2750 (1996); C. F. Chou, A. Jin, C. Y. Chao, S. W. Hui, C. C. Huang, and J. T. Ho, Phys. Rev. E 55, R6337 (1997).

[8] J. V. Selinger, J. Phys. (Paris) 49, 1387 (1988); R. Holyst, Phys. Rev. B 46, 15542 (1992).

[9] E. A. L. Mol, J. D. Shindler, A. N. Shalaginov, and W. H. de Jeu, Phys. Rev. E 54, 536 (1996); E. A. L. Mol, G. C. L. Wong, J.-M. Petit, F. Rieutord, and W. H. de Jeu, Physica B 248, 191 (1998).

[10] E. A. L. Mol, G. C. L. Wong, J.-M. Petit, F. Rieutord, and W. H. de Jeu, Phys. Rev. Lett. 79, 3439 (1997).

[11] R. Holyst, Phys. Rev. A 44, 3692 (1991); A. N. Shalaginov and V. P. Romanov, Phys. Rev. E 48, 1073 (1993).

[12] P. Mach et al., Langmuir 14, 4330 (1998).

[13] L. D. Landau and E. M. Lifshitz, Theory of Elasticity (Pergamon, Oxford, 1969) Chap. 2.

[14] M. Cagnon and G. Durand, Phys. Rev. Lett. 45, 1418 (1980).

[15] A. N. Shalaginov et al. (unpublished).

[16] J. L. Gallani, P. Martinoty, D. Guillon, and G. Poeti, Phys. Rev. A 37, 3638 (1988).

[17] R. Geer et al., Phys. Rev. Lett. 66, 1322 (1991). 